\title{
SOMATIC MARKERS AND EXPLICIT KNOWLEDGE ARE BOTH INVOLVED IN DECISION-MAKING
}

Shortened title: Somatic markers and decision-making

Sébastien Guillaume M.D., M.Sc. ${ }^{* a, b, c}$, Fabrice Jollant M.D., Ph.D. ${ }^{\text {a,b,c }}$, Isabelle Jaussent M.Sc., , Natalia Lawrence Ph.D ${ }^{\mathrm{e}}$, Alain Malafosse M.D., Ph.D. ${ }^{\text {b,d }}$, Philippe Courtet M.D., Ph.D. ${ }^{a, b, c}$

${ }^{\mathrm{a}}$ Montpellier 1 University, Montpellier F-34000, France

${ }^{\mathrm{b}}$ Inserm, U888, Montpellier F-34000, France

${ }^{c}$ Department of Psychological Medicine and Psychiatry, CHU Montpellier, F-34000, France.

${ }^{\mathrm{d} D e p a r t m e n t ~ o f ~ P s y c h i a t r y, ~ U n i v e r s i t y ~ H o s p i t a l ~ o f ~ G e n e v a, ~ C h e ̂ n e-B o u r g, ~ S w i t z e r l a n d ~}$

${ }^{\mathrm{e}}$ Wales Institute of Cognitive Neuroscience, School of Psychology, Cardiff University,.

\section{Corresponding author:}

Dr. S. Guillaume

Hôpital Lapeyronie

Service de Psychologie Médicale et Psychiatrie

371 avenue du Doyen G. Giraud

34295 Montpellier Cedex 5

France

Tel: +33 467338289; Fax: +33 467338988. E-mail: s-guillaume@chu-montpellier.fr 


\section{ABSTRACT}

In 1994, it was proposed that decision-making requires emotion-related signals, known as somatic markers. In contrast, some authors argued that conscious knowledge of contingencies is sufficient for advantageous decision-making. We aimed to investigate the respective roles of somatic markers and explicit knowledge in decision-making. Thirty healthy volunteers performed the Iowa Gambling Task (IGT). Conscious knowledge was assessed using a sensitive questionnaire and skin conductance responses (SCRs) were recorded. Most participants acquired a preference for advantageous choices during the task and generated larger anticipatory SCRs before disadvantageous relative to advantageous choices. Performance on the IGT and the autonomic response were positively correlated $(r=0.38$, $\mathrm{p}=0.045$ ). Moreover, there was a statistically significant difference in performance according to conscious awareness $(p=0.009)$. There was no significant association between level of explicit knowledge and SCR $(p=0.1)$. Finally, we did not find any interaction between explicit knowledge and performance although a lack of statistical power is not to be excluded. Advantageous decision-making therefore seems to be associated with two distinct, namely implicit and explicit, systems.

Keywords: decision-making, somatic marker hypothesis, Iowa Gambling Task, explicit Knowledge, learning 


\section{INTRODUCTION}

One of the most persistent challenges in daily life is to make decisions about possible courses of action. This topic has received much attention in a variety of separate disciplines ranging from neuroeconomics to psychology. Recently, it has been found than impairments in decision-making could play a part in many pathological behaviours in the field of neurology or psychiatry (Bechara, 2003; Jollant, et al., 2007). Moreover, investigations over the last decade have begun to see decision-making from the perspective of cognitive and affective neuroscience.

One influential conceptualization of decision-making is the somatic marker hypothesis (SMH) (Bechara, Damasio, Tranel, \& Damasio, 1997; Damasio, 1994) which proposes that decisionmaking is assisted by emotion-related signals. These so-called somatic markers, which develop with experience, may bias decisions away from a potentially disadvantageous choice, particularly in situations of complexity and uncertainty. The main support for this hypothesis comes from studies using the Iowa Gambling Task (IGT), which simulates real-life decisions in terms of uncertainty, reward and punishment (Bechara, 2003; Bechara, Damasio, Damasio, \& Lee, 1999). In this task, subjects have to make choices by picking cards from four decks which are either long-term advantageous or disadvantageous. Participants do not know at the beginning of the gamble which decks are advantageous and disadvantageous. In a major paper, Bechara, Damasio, Tranel, \& Damasio (1997) reported that healthy subjects decide advantageously before having a conscious knowledge of the advantageous choices. Furthermore, they showed larger anticipatory skin conductance responses (SCRs) prior to selecting a card from the disadvantageous decks compared to the advantageous decks. The authors concluded that somatic markers may assist the decision-making process in the IGT more than conscious knowledge. This was also supported by the findings of Carter \& SmithPasqualini (2004) who reported a positive correlation between performance and strength of 
the autonomic response (defined as the magnitude of SCRs before a bad choice minus the magnitude of response before a good choice) in a study of 30 healthy volunteers.

Nevertheless, in the last few years the SMH has been questioned (for a review (Dunn, Dalgleish, \& Lawrence, 2006)). The main criticism concerns the influence of conscious awareness on decision-making during the IGT. Indeed the IGT may be more cognitively penetrable than suggested by Bechara et al. (1997). Maia \& McClelland (2004) showed that the assessment of conscious awareness of the advantageous strategy during the IGT cannot be assessed by two simple open-ended questions as employed by Bechara et al (1997). Moreover, they found that this task could be correctly performed through access to conscious explicit knowledge. Therefore performance may be explained by consciously accessible knowledge rather than unconscious signals and performance does not necessarily require the generation of somatic markers. Moreover, they suggested that psychophysiological responses are caused by conscious knowledge. Unfortunately, they did not record SCRs in order to confirm their claim.

Furthermore, Gutbrod et al. (2006) measured behavioural performance and SCRs in patients with amnesia and in healthy controls. In controls, they found that the increase in anticipatory SCRs to disadvantageous choices occurred much later than the behavioural switch from bad to good decks. Moreover, they found no correlation between psychophysiological measures and performance. They demonstrated impaired IGT performance in amnesic patients and therefore suggested that the acquisition of a behavioural preference depends on the explicit memory of previous reinforcements encountered in the task. However, the small size of the control group $(\mathrm{N}=8)$ may explain this discrepancy and limit interpretation of these results.

Therefore, the respective roles of explicit knowledge and somatic markers in decision-making remains controversial. In the current study, we aimed to address this question. To this end, we assessed both SCRs following the methods of Bechara et al (1997), and measured explicit 
knowledge using similar questions and categorisations as Maia \& McClelland (2004). In view of the important roles previously attributed to both variables, we hypothesized that both explicit knowledge and somatic markers would be involved in decision-making without being linked to each other. 


\section{METHODS}

\section{Participants}

Thirty healthy subjects (11 men and 19 women) were recruited. Participants were interviewed using the Mini International Neuropsychiatric Interview (MINI 5.00) (Sheehan, et al., 1998). All subjects with a lifetime history of psychiatric disorders, neurological disorders, or on current medication (except oestroprogesterone contraception for woman) were excluded.

\section{Assessment of decision-making skills}

Decision-making skills were assessed using the computerized version of the IGT. The procedure was identical for all participants and has been described in detail elsewhere (Bechara, et al., 1999). Briefly, the subject has to make a choice between four decks of cards (60 cards each). When he or she clicks on a deck, he or she may win, or both win and lose money. The goal of the game is to win as much money as possible. The participant does not know that he or she will select a total of 100 cards. The subject also does not know that there are two advantageous decks for which little money is won but even less is lost (resulting in a net gain) and two disadvantageous decks for which a lot of money is won but even more is lost (resulting in a net loss).Howewer, they are informed that "some decks are better than others". The subject does not know when he or she will lose money, which creates a feeling of uncertainty. Recording of the choices is made automatically.

The 100 card selections were divided into five blocks of 20 cards and a total score. The scores represent the differences between the number of choices from the advantageous decks (C\&D) and the disadvantageous decks (A\&B).

\section{Assessment of conscious knowledge}


At the end of the game each participant was asked a list of questions. The questions were: (1)

tell me all you know about this game; (2) did you find any difference between the decks? (3) suppose you select 10 new cards from the deck $A / B / C / D$, will you on average win or lose money? (The question is repeated for each deck.); and (4) retrospectively, if you have to choose only one deck, which one will you choose in order to earn as much money as possible?

Assessment of level of knowledge was carried out as described by Maia \& McClelland (2004) into three levels of conscious knowledge: (1) level 0 : the participants do not have any conscious knowledge specifying a preference for one of the two best decks; (2) level 1: the participant has conscious knowledge specifying a preference for one of the two best decks but does not have conscious knowledge about the outcomes of the decks that could provide a basis for that preference; and (3) level 2: the participant has conscious knowledge specifying a preference for one of the two best decks and has conscious knowledge about the outcomes of the decks that could provide a basis for that preference.

\section{Skin conductance recording}

SCR activity was recorded during the IGT. The SCR data were acquired using an MP100WS system (BIOPAC Systems, Inc.) at a rate of 100 samples per second. The data were stored on a Macintosh computer and analyzed using Acknowledge 3.7 software for the MP100WS system

Electrodes were attached to the distal phalange of the first and second digits of the nondominant hand. To standardize the dermo-gel-electrode interface, participants were requested to wash their hands using a nonabrasive soap. Participants were then led into the testing room (adjacent) and sat on a comfortable chair. After the electrodes had been placed there was a 5min habituation period during which participants were asked to sit quietly, relax and move as 
little as possible. Two minutes of baseline SCRs were then recorded, during which there were no stimuli and participants were instructed not to move or talk.

Baseline SCR activity was assessed using three measurements per subject, recorded: (1) at rest; (2) in response to a handclap (delivered about $30 \mathrm{~cm}$ from the participant's left ear without warning); and (3) after a deep breath. These stimuli have been shown to reliably elicit SCRs in healthy individuals (Cacioppo \& Tassinary, 1991; Sierra, et al., 2002). In order to take into account the notoriously large individual differences in human SCRs, all psychophysiological measures were controlled for baseline activity.

The method for acquiring SCR data was identical to that used by Bechara et al. ( $1999 ; 1997)$ in their original studies. SCRs were recorded continuously throughout the task. They were divided into two categories: (1) anticipatory SCRs; and (2) outcome SCRs. Outcome SCRs were recorded during the 5 -sec interval after clicking on cards resulting in a gain and/or a penalty. Anticipatory SCRs, were defined as SCRs generated during the period between the end of the 5-sec interval of the outcome SCRs and the following selection of a deck. Inter-trial intervals were fixed at a minimum of $6-\mathrm{sec}$ in this version of the IGT to guarantee an anticipatory SCR period of at least 1 -sec prior to the next choice. There was no constraint on time for choice and most of the time anticipatory SCRs were very much longer than 1-sec (mean duration of anticipatory SCRs was $3.9 \mathrm{sec}$ in our sample).

Instructions on the screen during the choice period were "Please choose a card". Outcomes were given as "you win $\$ X$ " or "you lose $\$ Y$ ". Following the outcomes, participants were instructed to "please wait".

An investigator monitored the data recording. He also oversaw the reactions and movements of participants in order to identify artefact signals (i.e. a subject's movement). The investigator was positioned so as not to see the computer screen on which participants performed the IGT. Thus he was blind to the subjects' choices and performance. Maximal 
amplitudes (Micro Siemens) recorded during each time window for the two SCR categories were analyzed.

We analyzed the median maximal anticipatory amplitudes before either the advantageous or disadvantageous choices. Finally, we introduced a variable, named autonomic response, defined as the median maximal anticipatory SCR for the disadvantageous decks $(A+B)$ minus the median maximal anticipatory SCR scores for the advantageous decks $(C+D)$. The main hypothesis of this study concerned the relationship between anticipatory SCRs and IGT performance (Bechara, et al., 1997) so outcome SCRs was not further considered.

\section{Statistical analysis}

Statistical analyses were performed using SAS software, version 9.1 (SAS Institute, Cary, $\left.\mathrm{NC}^{\circ}, \mathrm{USA}\right)$.

The characteristics of the study population were described using median and range for quantitative variables with skewed distributions, mean and standard deviation (SD) for quantitative variables with normal distributions, and proportions for categorical variables. For continuous variables, the distributions were tested with Shapiro-Wilk's test.

Comparison of skewed distributions between two groups was performed using the MannWhitney rank sum test (two-group comparisons) and Kruskall-Wallis test (multiple-group comparisons). The Wilcoxon signed rank test was used to compare two dependent samples and the Friedman test for three or more dependent samples (e.g. separate groups of participants with different level of awareness). For the correlation analysis, Spearman's correlation and partial correlation coefficients were used. When comparisons were statistically significant, two by two comparisons were carried out, using Bonferroni's correction. 
Finally, as the IGT net score was normally distributed, the IGT net score was compared for comprehension level after adjustment for autonomic response, using an analysis of covariance.

For all comparisons, significance was set at $\mathrm{p}<0.05$. 


\section{RESULTS}

\section{Behavioural choice}

The mean IGT net score was 21.8 ( \pm SD 29.9). Figure 1 shows the acquisition of a preference for advantageous decks during the task. The performance scores between the different blocks of 20 choices were significantly different ( $<<0.0001$ ), with a continuous increase in IGT net score from choices 1 to 20 to choices 81 to 100 .

Performance significantly changed between the first (trials 1 to 50) and the second part of the task (trial 51 to 100) $(p=0.0015)$

\section{Psychophysiological measure}

Median anticipated SCR amplitudes recorded before the disadvantageous choices were significantly higher than those recorded before the advantageous choices (respectively 0.025 $\mu$ siemens $[0.007-0.181]$ and $0.020 \mu$ siemens $[0.007-0.098](\mathrm{p}=0.0006)$. The median difference between these two amplitudes (i.e. the autonomic response) was 0.001 [-0.021 $0.088]$.

Autonomic response also changed along the task with a significant increase between the first and the second part of the task $(p=0.04)$.

\section{Conscious knowledge}

At the end of the game, 14 of the 30 subjects (46.7\%) reached level 2 (full knowledge), 7 $(23.3 \%)$ reached level 1 and $9(30 \%)$ failed to understand anything about the correct strategy (level 0) (see Figure 2). This is a comparable spread of data to previous reports (Lawrence, Jollant, O'Daly, Zelaya, \& Phillips, 2008). 


\section{Relationship between performance, autonomic response and conscious knowledge}

Performance in the IGT and autonomic response were positively correlated $(r=0.38, p=0.045)$ : the greater the difference between anticipatory SCRs before advantageous and disadvantageous choices, the better the final performance. IGT net score was not correlated with anticipatory SCRs before advantageous choices only or before disadvantageous choices only.

There was a statistically significant difference in median performance according to comprehension level $(\mathrm{p}=0.009)$ (Figure 2). The median net score was 45 [-10-66] for level 2, $20[-6-56]$ for level 1 , and $-2[-48-28]$ for level 0 . Post hoc analyses revealed a significant difference between level 2 and level 0 in IGT performance. To increase the statistical power, we combined participants from levels 0 and 1 of explicit knowledge. Regrouping participants into two groups (level 2 vs. level $0+1$ ) did not modify the association between explicit knowledge and performance. We found a median performance of 45 [-10-66] for the level 2 group and $3[-48-56]$ for the level $0+1$ group $(\mathrm{p}=0.006)$.

There was no statistically significant association between level of explicit knowledge and autonomic response $(\mathrm{p}=0.1)$ : median SCRs were 0.0072 [- $0.0005-0.0883]$ for level 2, and $0.0007[-0.0212-0.0621]$ for level $0+1$.

Finally, autonomic response did not significantly modify the relationship between explicit knowledge and IGT net score assessed using analysis of covariance. 


\section{DISCUSSION}

The results of this study show that: (1) performance in the IGT was correlated with autonomic response i.e. the difference between anticipatory SCRs before advantageous and disadvantageous choices, but not with anticipatory SCRs before advantageous or disadvantageous choices only; (2) performance in the IGT was associated with conscious knowledge about the underlying contingencies; and (3) Conscious knowledge and anticipatory SCRs do not seem to be associated. Our results assessing the respective impact of autonomic response and explicit knowledge in performance during the IGT therefore reveal new information.

First, our results support the involvement of somatic markers in decision-making. They are also in agreement with two previous studies that also found a positive correlation between strength of the autonomic signal and performance during the IGT (Carter \& Smith-Pasqualini, 2004; Oya, et al., 2005) but not between anticipatory SCRs before a disadvantageous choice and performance (Dunn, et al., 2006). Therefore, in contrast to what was initially proposed by Damasio et al. (1994), it is not so much the absolute intensity of the signal (SCRs) before the disadvantageous choice that is important, but the contrast between this signal and that developed before the advantageous choices. Another line of evidence comes from fMRI experiments. Critchley et al. (Critchley, Elliott, Mathias, \& Dolan, 2000) confirmed the contribution of ventral and medial prefrontal regions to SCR generated during a gambling task. These brain regions are close to those implicated in the IGT. Recently, two fMRI studies have found that IGT performance was positively correlated with activation during disadvantageous relative to advantageous choices, in the medial frontal gyrus (BA10) (Fukui, Murai, Fukuyama, Hayashi, \& Hanakawa, 2005; Lawrence et al., 2008) and the left orbitofrontal cortex (BA47) (Lawrence, et al., 2008). Therefore, these studies suggest that the 
differential neural response to choices from disadvantageous relative to advantageous decks is the key variable associated with successful performance on the IGT. The differential autonomic response reported here could be a reflection of this activation shown in fMRI.

Second, our results are compatible with the data of Maia \& McClelland (2004) showing the involvement of explicit knowledge in performance during the IGT. Healthy individuals with higher levels of understanding also have better performance. The influence of explicit knowledge may therefore be more important than previously stated by Bechara et al. (Bechara, et al., 1997)

Third, questions persist about the meaning of this psychophysiological response during the IGT. Bechara et al. $(2003 ; 1997)$ have interpreted these anticipatory SCRs as reflecting somatic signals that could sufficiently guide advantageous behaviour. Maia \& McClelland (2004) proposed that SCRs are caused by conscious knowledge of the situation. Our findings suggest that SCRs and knowledge are not associated with each other. Decision-making during the IGT may therefore be guided by two different and distinct pathways. Yet, both distinct systems could interact to guide decision-making. We did not find any association between both pathways on IGT performance. However, a lack of statistical power may have hindered these findings. For instance, it is possible that explicit knowledge may be useful to guide adequate decision-making only if adequate somatic markers are available. This would explain why some patients with ventromedial prefrontal cortex lesions and weak anticipatory SCRs have poor performance in spite of correct explicit understanding (Bechara, Damasio, Tranel, \& Damasio, 2005). This has to be tested.

Another criticism levelled at the SMH concerns the evolution of anticipatory autonomic responses and explicit knowledge along the course of the task. For instance, Gutbrod et al. (2006) suggested that the behavioural preference for advantageous decks precedes the emergence of the autonomic response. If our results show parallel changes in behavioural 
performance and autonomic response between the first and the second part of the task, we have deliberately not assessed this relationship, because we believe that the structure of the IGT prevents a dynamic analysis of psychophysiological data along the course of the task. Firstly, to be sufficiently sensitive, the evolution is calculated per blocks of 20 cards, this severely limits the number of SCR measures prior to choosing from each deck. Furthermore, in some trials participants exclusively selected cards from advantageous or disadvantageous decks resulting in missing data for some anticipatory SCRs measures. Finally, after a certain time playing some subjects systematically avoided some decks. All of these elements lead to measurement bias making a valid statistical analysis of the dynamic interaction between the autonomic response and explicit knowledge during the IGT extremely difficult. This important question should therefore be addressed with more optimal experimental designs.

Our study has several limitations. First is the correlational aspect of the link. This approach does not allow causal conclusions. Another limitation is the structure of the IGT. As suggested previously, we think that it is very difficult to properly investigate dynamic evolution of the interaction between autonomic response and performance using this task. The third limitation is due to our assessment of explicit knowledge. It has been suggested that the questionnaire used by Maia \& McClelland (2004) to question the participants on their explicit understanding may have clued in subjects and made them aware (Persaud, McLeod, \& Cowey, 2007). In addition, unlike several authors (Cella, Dymond, Cooper, \& Turnbull, 2007; Evans, Bowman, \& Turnbull, 2005; Maia \& McClelland, 2004) explicit knowledge was assessed once at the end of the IGT and it has been argued that such post-hoc measures lack sufficient sensitivity to detect ongoing changes in behavioural performances during the task (Lovibond \& Shanks, 2002)..These could lead to a bias in our correlation between prior performance and measures of participants' conscious awareness obtained post-experimentally. 
A study with a similar hypothesis but with a different and concurrent assessment of awareness (i.e: (Bowman, Evans, \& Turnbull, 2005)) could overcome this potential bias.

Fourth, we cannot exclude the possibility that the association between performance and autonomic response was a reflection of the higher variance of the deck rather than of the negative outcome as proposed by Tomb et al. (Tomb, Hauser, Deldin, \& Caramazza, 2002). These authors rely on the variant task of the IGT, where the good decks have the highest variance in reward and punishment, and found higher SCRs for the advantageous decks. In response, Bechara and al. suggest that in the original IGT the SCRs perhaps reflect a negative somatic state associated with bad outcomes and in the modified IGT, they reflect a positive somatic state associated with good outcomes. SCR is a simple and well-validated measure of emotional activity (Grillon, 2004). However, it gives no information on emotional valence and does not allow discrimination between positive and negative markers.

Fifth, as already outlined, a lack of statistical power due to the small sample size may have hindered an association between the autonomic response and explicit knowledge, or the interactive effect of both variables on the IGT performance. The final limitation of the study is the mix of males and females in our sample. Gender ratios have been shown to be important when measuring decision-making (Bolla, Eldreth, Matochik, \& Cadet, 2004). Nevertheless, complementary analysis in our population showed no difference between males and females in term of performance, explicit knowledge or SCR.

In conclusion, our results suggest that decision-making during the IGT may be guided by two pathways, an explicit and an implicit one, which do not appear to be linked to each other. The question remains whether both systems may influence each other in modifying decisionmaking. Future research should also aim at understanding the basis of the individual differences in decision-making, exploring factors such as affective reactivity (Peters and Slovic, 2000), interoceptive awareness (Critchley, Wiens, Rotshtein, Ohman, \& Dolan, 2004), 
education (Evans, Kemish, \& Turnbull, 2004) and thinking style (Epstein, Pacini, Denes-Raj, \& Heier, 1996). This would help to refine existing theories about human decision-making. 


\section{ACKNOWLEDGEMENTS}

This study received financial support from CHU Montpellier (PHRC UF 7653). Dr S. Guillaume received a grant from the Fondation pour la Recherche Médicale (Action Dynamique en Psychiatrie 2004). We would like to thank Camille Laurent for data collection. 


\section{REFERENCES}

Bechara, A. (2003). Risky business: emotion, decision-making, and addiction. J Gambl Stud, 19(1), 23-51.

Bechara, A., Damasio, H., Damasio, A. R., \& Lee, G. P. (1999). Different contributions of the human amygdala and ventromedial prefrontal cortex to decision-making. $J$ Neurosci, 19(13), 5473-5481.

Bechara, A., Damasio, H., Tranel, D., \& Damasio, A. R. (1997). Deciding advantageously before knowing the advantageous strategy. Science, $275(5304), 1293-1295$.

Bechara, A., Damasio, H., Tranel, D., \& Damasio, A. R. (2005). The Iowa Gambling Task and the somatic marker hypothesis: some questions and answers. Trends Cogn Sci, 9(4), 159-162; discussion 162-154.

Bolla, K. I., Eldreth, D. A., Matochik, J. A., \& Cadet, J. L. (2004). Sex-related differences in a gambling task and its neurological correlates. Cereb Cortex, 14(11), 1226-1232.

Bowman, C. H., Evans, C. E., \& Turnbull, O. H. (2005). Artificial time constraints on the Iowa Gambling Task: the effects on behavioural performance and subjective experience. Brain Cogn, 57(1), 21-25.

Cacioppo, J., \& Tassinary, L. (1991). Principles of psychophysiology. Cambridge: Cambridge university press.

Carter, S., \& Smith-Pasqualini, M. C. (2004). Stronger autonomic response accompagnies better learning: a testof Damasio's somatic markers hypothesis Cognition and Emotion, 18(7), 901-911.

Cella, M., Dymond, S., Cooper, A., \& Turnbull, O. (2007). Effects of decision-phase time constraints on emotion-based learning in the Iowa Gambling Task. Brain Cogn, 64(2), 164-169.

Critchley, H. D., Elliott, R., Mathias, C. J., \& Dolan, R. J. (2000). Neural activity relating to generation and representation of galvanic skin conductance responses: a functional magnetic resonance imaging study. J Neurosci, 20(8), 3033-3040.

Critchley, H. D., Wiens, S., Rotshtein, P., Ohman, A., \& Dolan, R. J. (2004). Neural systems supporting interoceptive awareness. Nat Neurosci, 7(2), 189-195.

Damasio, A. R. (1994). Descartes' error: emotion,reason, and the human brain (Putnam ed.). New York.

Dunn, B. D., Dalgleish, T., \& Lawrence, A. D. (2006). The somatic marker hypothesis: a critical evaluation. Neurosci Biobehav Rev, 30(2), 239-271.

Epstein, S., Pacini, R., Denes-Raj, V., \& Heier, H. (1996). Individual differences in intuitive-experiential and analytical-rational thinking styles. J Pers Soc Psychol, $71(2), 390-405$.

Evans, C. E., Bowman, C. H., \& Turnbull, O. H. (2005). Subjective awareness on the Iowa Gambling Task: the key role of emotional experience in schizophrenia. J Clin Exp Neuropsychol, 27(6), 656-664.

Evans, C. E., Kemish, K., \& Turnbull, O. H. (2004). Paradoxical effects of education on the Iowa Gambling Task. Brain Cogn, 54(3), 240-244.

Fukui, H., Murai, T., Fukuyama, H., Hayashi, T., \& Hanakawa, T. (2005). Functional activity related to risk anticipation during performance of the Iowa Gambling Task. Neuroimage, 24(1), 253-259.

Grillon (2004). Methods of affective clinical psychophysiology. In O. u. press (Ed.), Neurobiology of mental illness (second ed., pp. 127-140): Charney. 
Gutbrod, K., Krouzel, C., Hofer, H., Muri, R., Perrig, W., \& Ptak, R. (2006). Decisionmaking in amnesia: do advantageous decisions require conscious knowledge of previous behavioural choices? Neuropsychologia, 44(8), 1315-1324.

Jollant, F., Guillaume, S., Jaussent, I., Bellivier, F., Leboyer, M., Castelnau, D., et al. (2007). Psychiatric diagnoses and personality traits associated with disadvantageous decision-making. Eur Psychiatry, 22(7), 455-461.

Lawrence, N. S., Jollant, F., O'Daly, O., Zelaya, F., \& Phillips, M. L. (2008). Distinct Roles of Prefrontal Cortical Subregions in the Iowa Gambling Task. Cereb Cortex.

Lovibond, P. F., \& Shanks, D. R. (2002). The role of awareness in Pavlovian conditioning: empirical evidence and theoretical implications. J Exp Psychol Anim Behav Process, 28(1), 3-26.

Maia, T. V., \& McClelland, J. L. (2004). A reexamination of the evidence for the somatic marker hypothesis: what participants really know in the Iowa gambling task. Proc Natl Acad Sci U S A, 101(45), 16075-16080.

Oya, H., Adolphs, R., Kawasaki, H., Bechara, A., Damasio, A., \& Howard, M. A., 3rd (2005). Electrophysiological correlates of reward prediction error recorded in the human prefrontal cortex. Proc Natl Acad Sci U S A, 102(23), 8351-8356.

Persaud, N., McLeod, P., \& Cowey, A. (2007). Post-decision wagering objectively measures awareness. Nat Neurosci, 10(2), 257-261.

Peters, E., \& Slovic, P. (2000). The springs of action: Affective and analytical information processing in choice. Personality and Social Psychology Bulletin, 26, $1465-1475$.

Sheehan, D. V., Lecrubier, Y., Sheehan, K. H., Amorim, P., Janavs, J., Weiller, E., et al. (1998). The Mini-International Neuropsychiatric Interview (M.I.N.I.): the development and validation of a structured diagnostic psychiatric interview for DSM-IV and ICD-10. J Clin Psychiatry, 59 Suppl 20, 22-33; quiz 34-57.

Sierra, M., Senior, C., Dalton, J., McDonough, M., Bond, A., Phillips, M. L., et al. (2002). Autonomic response in depersonalization disorder. Arch Gen Psychiatry, 59(9), 833-838.

Tomb, I., Hauser, M., Deldin, P., \& Caramazza, A. (2002). Do somatic markers mediate decisions on the gambling task? Nat Neurosci, 5(11), 1103-1104; author reply 1104. 